\title{
Unusual manifestations of craniofacial fibrous dysplasia: clinical, endocrinological and computed tomographic features
}

\author{
B.D. Daly, C.C. Chow ${ }^{1}$ and C.S. Cockram ${ }^{1}$ \\ Departments of Diagnostic Radiology and Organ Imaging and ${ }^{l}$ Medicine, Chinese University of Hong \\ Kong, Prince of Wales Hospital, Shatin, N.T., Hong Kong
}

\begin{abstract}
Summary: Clinical, endocrinological and computed tomographic features of three patients with unusual manifestations or complications of craniofacial involvement of fibrous dysplasia are presented. One patient with polyostotic fibrous dysplasia presented in late pregnancy with acute onset of bilateral optic nerve compression and blindness secondary to a rapidly expanding mass of fibrous dysplasia tissue involving the sphenoid, pituitary and optic chiasm regions. A second patient with polyostotic fibrous dysplasia developed thyrotoxicosis and probable gigantism/acromegaly in keeping with a rare form of McCune-Albright syndrome. Extensive bony distortion of the skull and facial bones by fibrous dysplasia made clinical recognition of these complications more difficult. A third patient had monostotic fibrous dysplasia with marked sclerosis of the sphenoid bone on plain radiographs which mimicked appearances of a meningioma and resulted in a negative craniotomy as computed tomography was not yet available at the time of presentation.

Each case demonstrated rare complications of craniofacial fibrous dysplasia and highlighted the wide spectrum of appearances in which it may manifest, often resulting in overlap and diagnostic confusion with other disease processes. The value of computed tomography in assessment is emphasized.
\end{abstract}

\section{Introduction}

Fibrous dysplasia is an idiopathic disorder of skeletal bone in which medullary bone is replaced by fibro-osseous tissue leading to bony distortion, overgrowth and fractures. The disease occurs in monostotic and polyostotic forms, and the latter may be complicated rarely by skin pigmentation and multiple endocrinopathies (McCune-Albright Syndrome).$^{1-4}$ Twenty per cent of monostotic and $40-60 \%$ of polyostotic cases involve the skull and face. ${ }^{5}$ The characteristic lion face or 'Leontiasis Ossea' may develop if the facial bones are extensively involved in the disease process. Bone expansion may encroach on the paranasal sinuses, nasal fossae, orbits and neurovascular canals. ${ }^{6}$ In the calvarium and mandible involvement occurs most commonly in the form of multiple cystic lesions whereas in the base of the skull, thickening and sclerosis of bone is the usual finding.?

The radiological appearances are variable and depend upon the amount of bone laid down in affected areas. Appearances range from very

Correspondence: B.D. Daly, Radiology Department, University of Maryland Hospital, 22 South Greene Street, Baltimore MD 21201, USA.

Accepted: 6 August 1993 radiolucent lesions composed of mainly fibrous tissue to markedly sclerotic expanded bone made up of dense bony trabeculae with little fibrous element. ${ }^{8}$ Any degree between these two extremes may be seen and craniofacial fibrous dysplasia (CFFD) may thus mimic a wide range of other body pathologies. Computed tomography (CT) is a particularly helpful technique for the assessment of CFFD involvement of the facial bones and skull base because of its ability to image dense bony structures accurately.

The clinical features, CT appearances and relevant laboratory findings are presented in three patients who presented to our hospital with rare manifestations or complications of CFFD. The value of CT in the assessment of these unusual problems is emphasized.

\section{Case reports}

Patient 1

A 27 year old Chinese woman first presented one week prior to the delivery of her first child when she suddenly became blind in the right eye. This was preceded by blurring of vision and headaches for a 
few days. On examination she was noted to have hypertelorism and marked distortion of the normal anatomy of the skull bones. A large 'cafe-au-lait' pigmented area with an irregular border was noted over the right side of the face and did not cross the midline. The right eye was totally blind with no perception of light and evidence of optic atrophy on ophthalmoscopy. The consensual light reflex was present in the right eye while the direct light reflex was lost.

A prolactin concentration measured 4 days after delivery had been $9,500 \mathrm{mU} / 1$ (normal $<800 \mathrm{mU} /$ l) but subsequently fell to normal. She did not breast feed. Pituitary function was otherwise normal at this time. Radiographs showed a cystic lesion in the left fourth rib and gross thickening and distortion of all skull bones characteristic of polyostotic fibrous dysplasia. A CT head scan carried out on the day of delivery confirmed marked changes of fibrous dysplasia of bone. In addition there was evidence of a $2.1 \mathrm{~cm}$ diameter soft tissue 'tumour' in the pituitary fossa extending anteriorly and to the right of the sella turcica with associated bone erosion. Treatment with bromocriptine was commenced on an empirical basis as a prolactin-secreting pituitary tumour could not be excluded. Surgery was considered too hazardous due to the marked bony thickening. At this time vision in the left eye was normal. The patient was discharged with close outpatient follow-up.

She was readmitted to hospital as an emergency 8 weeks later with a one week history of blurred vision in the left eye. The right eye remained totally blind. On examination the visual acuity in the left eye was reduced to counting fingers at 6 feet. The direct light reflex in the left eye remained normal. A diagnosis of chiasmal compression was made. Repeat CT head scan showed considerable enlargement of the 'tumour' compared to 2 months earlier. A poorly defined $3.5 \mathrm{~cm}$ enhancing mass occupied the entire sphenoid sinus and pituitary fossa with extension to the suprasellar region, right orbital apex and petrous tip (Figure 1). Destruction of the sella, planum sphenoidale, clivus, posterior ethmoids and part of greater sphenoid wing bilaterally was also seen on bone window images (Figure 2). The CT changes were considered most likely due to an aggressive tumour of uncertain origin. An exploratory transfrontal craniotomy revealed a soft extradural mass overlying the right optic canal and extending medially to the planum sphenoidale and compressing the optic nerves. Profuse bleeding occurred and only small fragments of soft bluish tissue were biopsied.

Slow improvement in sight occurred over the next 4 months with useful navigational vision and an ability to count fingers at 3 feet. In view of the very rapid progression of the soft tissue lesion, together with doubts regarding the histology, post-

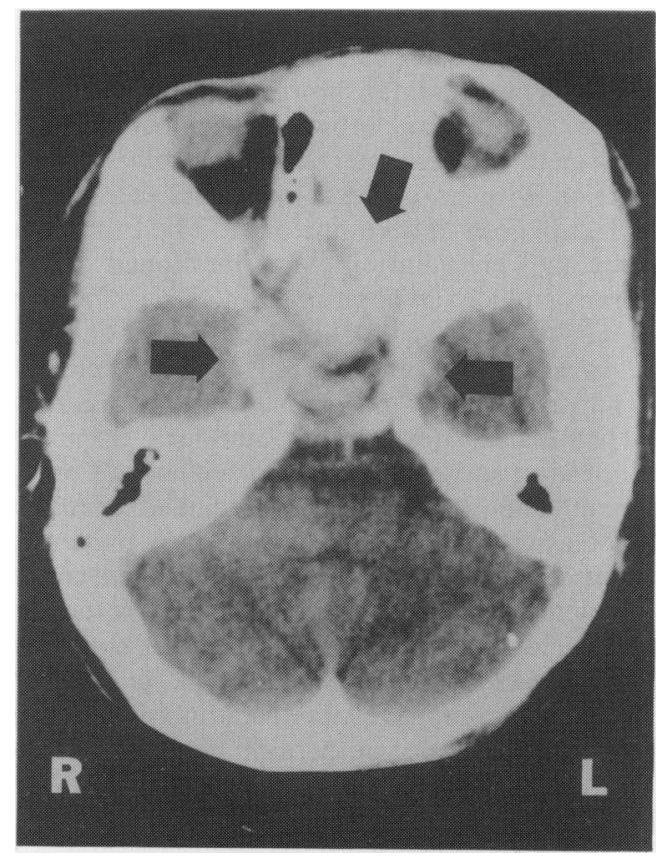

Figure 1 Patient 1: contrast-enhanced axial CT scan. Enhancing soft tissue fibrous dysplasia mass occupies sphenoid, clivus, sella and parasellar regions (black arrows). Distorted skull shape also due to fibrous dysplasia.

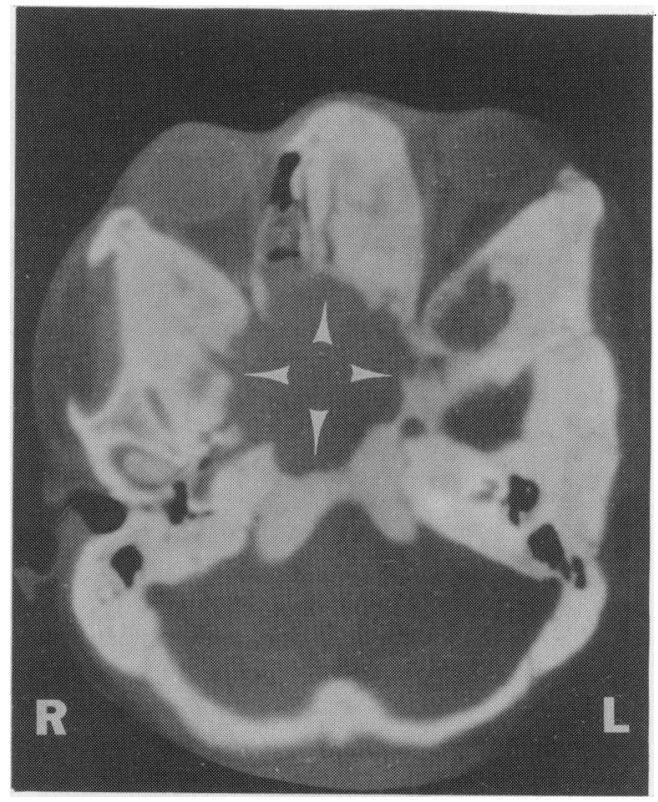

Figure 2 Patient 1: axial CT scan. Bone window image at lower level than Figure 1. The tumour has eroded the sphenoid, clivus and ethmoid bones (white arrowheads). All the bones (excepting the left occiput) are thickened and sclerosed by fibrous dysplasia. 
operative radiotherapy was given to the pituitary region. A CT scan carried out following radiotherapy 2 months later showed no further progression of the tumour. Subsequent endocrinological assessment one year later showed normal pituitary function with no evidence of excess secretion of prolactin, growth hormone or ACTH. Four years following presentation she developed radiotherapy-induced hypopituitarism. Serial CT scans showed progressive involution of the tumour mass following irradiation and only a small pituitary gland was detected in the sellar region (Figure 3).

Histological review of 'tumour' fragments removed at surgery showed no evidence of malignancy. The findings noted included extensive fibroblastic proliferation, granulation tissue and thin vascular channels. These findings were consistent with proliferation of fibrous dysplasia, and together with the clinical progress were consistent with rapid expansion of fibrous dysplasia commencing in late pregnancy and continuing into the immediate post-partum period.

\section{Patient 2}

A 14 year old Chinese male was referred for assessment of chronic right hip pain and intermittent palpitations. He was known to have fibrous dysplasia confirmed histologically since the age of 9. He had no other past or family history. On examination, asymmetrical coarse facial features with prominent supraorbital ridges, a broad nose and thick lips were noted. No abnormal skin pigmentation could be found. He was in advanced puberty (Tanner stage 4) with a weight of $51.5 \mathrm{~kg}$ and height $1.72 \mathrm{~m}$. He appeared thyrotoxic with fine hand tremor, sinus tachycardia and diffuse thyroid enlargement with bruit. There was no infiltrative ophthalmopathy nor dermopathy. Visual fields were normal.

Serum alkaline phosphatase was grossly elevated at $1103 \mathrm{IU} / 1$ (normal 130-525), with normal calcium and phosphate concentrations. Thyroid function tests confirmed hyperthyroidism: thyroid stimulation hormone $<0.02 \mathrm{mIU} / 1$ (normal $0.3-$ 4.0); and free triiodothyronine $13.7 \mathrm{pmol} / 1$ (normal 3.3-8.2). Thyroid anti-thyroglobulin and antimicrosomal antibodies were negative. Baseline gonadol hormonal evaluation included a testosterone level of $9.4 \mathrm{nmol} / \mathrm{l}$ (normal male adult 12.0-34.0), luteinizing hormone (LH) $1.8 \mathrm{IU} / 1$ (normal male adult $<15$ ), follicle stimulating hormone (FSH) $5.9 \mathrm{IU} / 1$ (normal male adult $<8$ ) and prolactin $375 \mathrm{mIU} / 1$ (normal <480). Table I shows results of a gonadotrophin-releasing hormone (GnRH) test which demonstrates subnormal basal gonadotrophins and a slightly subnormal response of FSH and LH to the injection of GnRH. Table II shows non-suppressibility of growth hormone by oral glucose. Exaggerated release of

Table I Effect of gonadotropin-releasing hormone (GnRH) on gonadotropin levels

\begin{tabular}{lccc}
\hline & \multicolumn{3}{c}{ Time (minutes) } \\
& 0 & 20 & 60 \\
\hline FSH (IU/1) & 4.9 & 11.0 & 11.8 \\
LH $(\mathrm{IU} / \mathrm{l})$ & $<1.8$ & 20.4 & 17.5 \\
\hline
\end{tabular}

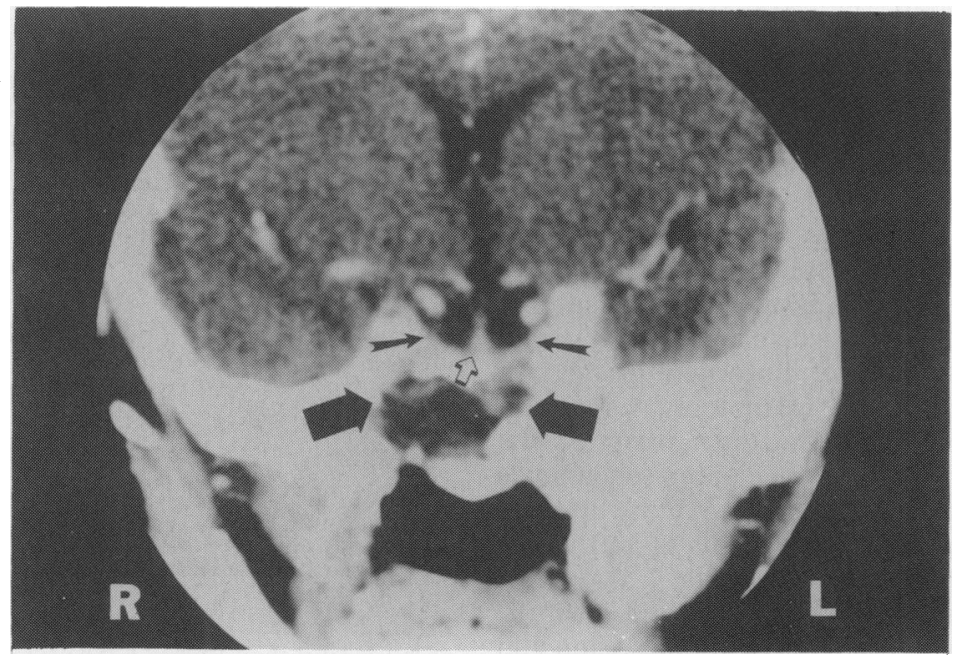

Figure 3 Patient 1: contrast-enhanced coronal CT scan. Scan done 15 months after images 1 and 2 shows normal pituitary gland (black arrowhead) within enlarged CSF-filled sella (small black arrows). Residual enhancing fibrous dysplasia soft tissue in the sphenoid region only (large black arrows). 
Table II Growth hormone concentrations during a $75 \mathrm{~g}$ oral glucose tolerance test in both the thyrotoxic and euthyroid state

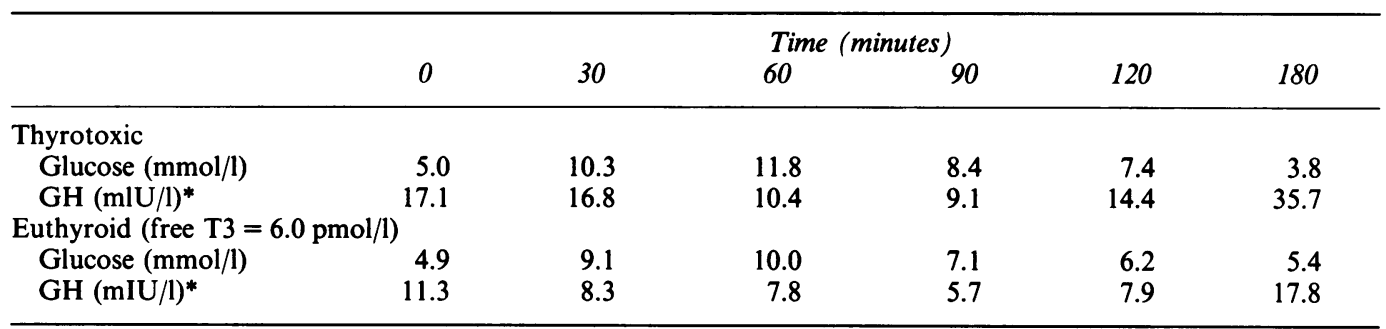

*Normal $<2 \mathrm{mIU} / 1$.

growth hormone in response to thyrotrophinreleasing hormone (TRH) with a rise to $>60 \mathrm{mIU} / 1$ at 20 and 60 minutes following intravenous injection of $200 \mu \mathrm{g}$ of TRH was noted. Radiological evaluation revealed polyostotic fibrous dysplasia involving multiple bones. CT confirmed bilateral asymmetrical sclerotic thickening involving almost all of the calvarium and facial bones and also noted a small pituitary fossa due to overgrowth of the clivus and sphenoid bones. No pituitary tumour was detected (Figure 4). The frontal sinuses were enlarged. Softening of the abnormal bone within the occiput resulted in basilar invagination with the odontoid peg lying above the hard palate-occiput (Chamberlains) line (Figure 5). Thyrotoxicosis and probable gigantism in association with polyostotic fibrous dysplasia were diagnosed.

Alkaline phosphatase has remained elevated (up to $1,915 \mathrm{IU} / \mathrm{l})$ with normal serum calcium and phosphate despite control of hyperthyroidism with carbimazole. Serum parathyroid hormone by radioimmunometric assay has been elevated at $9.7 \mathrm{pmol} / 1$ (normal 1.16-5.67). No specific treatment of gigantism/acromegaly has been instituted to date.

\section{Patient 3}

A 65 year old Chinese woman was referred for investigation because of slowly progressive reduction of visual acuity in both eyes. She had had cranial surgery for suspected meningioma 20 years previously, though only 'fibrous tissue' was obtained at craniotomy. The presence of marked bony sclerosis on skull radiographs and a history of headache was interpreted at that time as likely due

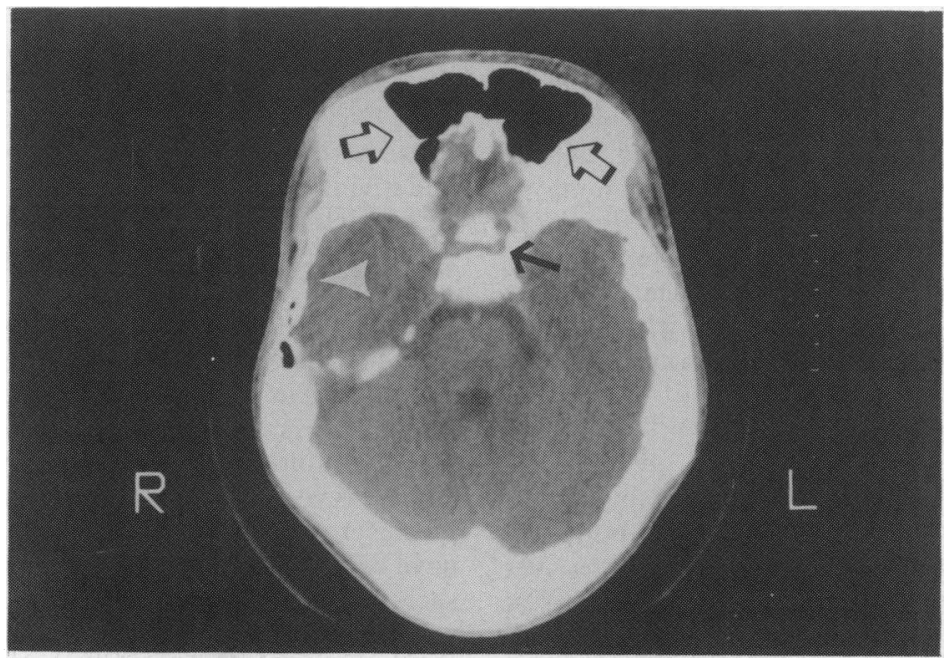

Figure 4 Patient 2: axial CT scan. Sclerotic thickening of fibrous dysplasia in all skull bones except part of right squamous (white arrowhead). No evidence of pituitary adenoma within small sella (black arrow). Large frontal sinuses are possibly due to coexistent acromegaly (open arrows). 


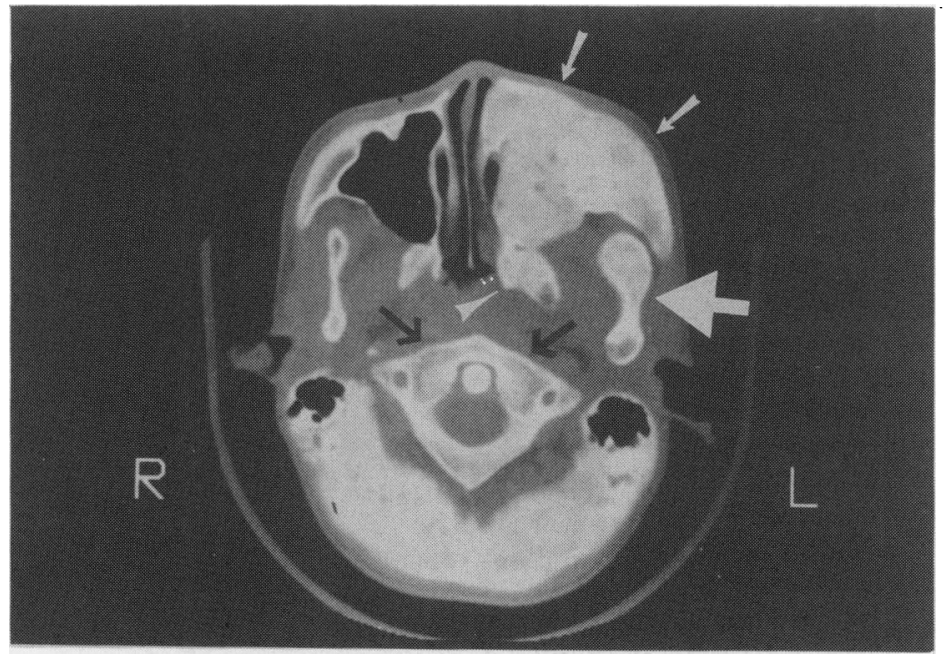

Figure 5 Patient 2: axial CT scan. Dense sclerotic fibrous dysplasia changes in expanded left maxilla (small white arrows), left pterygoid (white arrowhead), left mandible (large white arrow) and occiput. Left maxillary $2 \eta$ trum is obliterated. Atlas and dens lie in high position due to fibrous dysplasia-induced softening of occipital bone with resultant basilar invagination (black arrows).

to a sphenoid meningioma. Apart from deterioration in visual acuity, she had been asymptomatic in the intervening two decades.

On examination, there was mild proptosis of the left eye with associated prominence of the supraorbital region. There was evidence of an old left temporal craniotomy scar. Visual acuity was bilaterally $6 / 18$. Physical examination was otherwise normal. Skull and facial radiographs showed marked sclerosis of the greater wing of the left sphenoid bone (Figure 6). CT scan showed extensive sclerotic bony thickening of the greater wing of the left sphenoid extending into the left frontal, zygomatic and pterygoid bones also (Figure 7). Postoperative appearances were noted at the craniotomy site. No evidence of meningioma or other tumour was shown. The brain appeared normal. The CT appearances were interpreted as typical of CFFD. No further investigations were done. Her visual acuity was treated with corrective lenses and she remains well on follow-up.

\section{Discussion}

The ability of CT to resolve detail of both soft tissue and dense bony structures makes it a very suitable imaging modality for the assessment of CFFD. While magnetic resonance imaging (MRI) provides more detailed soft tissue images and is more sensitive to pathological change than CT, it produces a low signal from bone affected by fibrous dysplasia which may be difficult to interpret, especially if dense sclerotic change has occurred. ${ }^{6}$

The development of a rapidly enlarging 'tumourous' form of fibrous dysplasia in the skull base causing visual disturbance during pregnancy is very rare. We know of only one previously documented case which occurred in the same patient during two separate pregnancies. ${ }^{9}$ As in our case the lesion appeared as an enhancing soft tissue mass in the sphenoid region on CT. Unlike our case it was possible to remove the mass surgically with restoration of eyesight. It is recognized as unusual for fibrous dysplasia lesions to grow rapidly after skeletal maturation has occurred ${ }^{6}$ and it would seem likely, as suggested by Okudera et al..${ }^{9}$ that some hormonal change during pregnancy is the underlying cause. In our patient the large size and very vascular nature of the CFFD tumour precluded surgical removal and irradiation therapy was substituted. Irradiation therapy was considered necessary in this case due to initial doubts regarding the histopathological nature of the tumour and the urgent need to relieve optic nerve compression. CT was helpful in the demonstration of the size and infiltrative nature of the lesion and of its compressive effects on the optic nerves and chiasm. However, on CT criteria alone, the 'tumour' could have been due to an aggressive pituitary neoplasm such as a prolactinoma or even a sarcoma arising within fibrous dysplasia tissue (Figures 1 and 2). While sarcomas develop with increased frequency in patients with fibrous dysplasia, most occur in patients who have had previous irradiation.

Our second case demonstrates a rare form of 


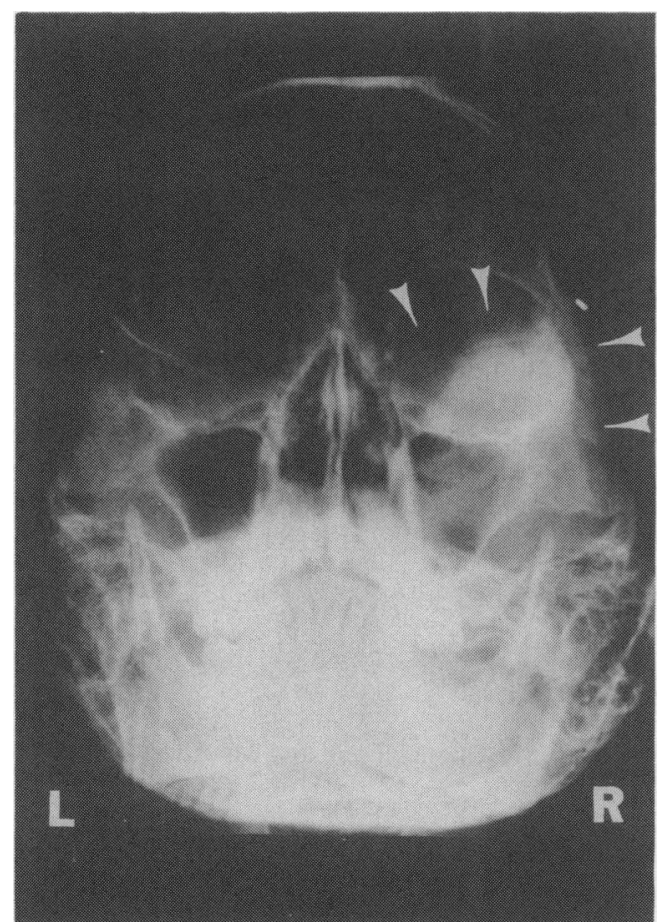

Figure 6 Patient 3: Water's view. Fibrous dysplasia with dense sclerosis of left sphenoid wing mimicking a hyperostotic meningioma (white arrows). Clip at old craniotomy scar.
McCune-Albright syndrome with the association of CFFD, and both hyperthyroidism and probable gigantism/acromegaly. It is interesting to note the absence of pigmented skin rash. Very few cases of growth hormone disorder complicating fibrous dysplasia have been documented. Reviews of the literature by Lipson and $\mathrm{Hsu}^{10}$ and Cutler et al. ${ }^{11}$ revealed six and 17 cases, respectively, of welldocumented clinical and biochemical acromegaly. The distortion of facial features caused by the bony deformities of CFFD leading to confusion with acromegaly has also been recognized. ${ }^{12}$ In our patient, biochemical studies point strongly towards a diagnosis of acromegaly in addition to proven evidence of hyperthyroidism. His facial appearances could be due in part either to CFFD or acromegaly though the asymmetrical left-sided enlargement of maxilla and mandible favours CFFD as the major cause of bone thickening (Figure 5). Large frontal sinuses may be due to acromegaly, however (Figure 4). The pituitary gland itself was not well seen due to much artefact on both axial and coronal cuts caused by very thick adjacent bone and this is a well-recognized limitation of CT. A growth hormone-secreting adenoma possibly in an ectopic suprasellar or hypothalamic position could not be excluded and should be sought with an MRI scan (currently unavailable). Soft tissues are not degraded by thick bone on MRI even though the bone itself is poorly demonstrated. While currently asymptomatic the presence of

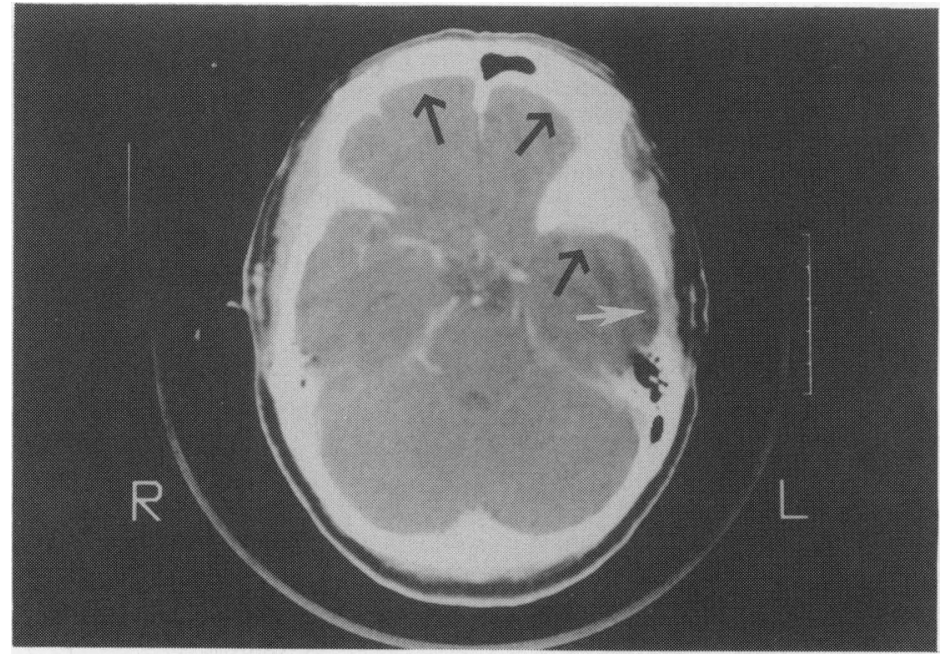

Figure 7 Patient 3: contrast-enhanced axial CT scan. Sclerotic thickening of left sphenoid and frontal bones typical of fibrous dysplasia (black arrows) also extended into the pterygoid plate and zygoma (not shown). No evidence of meningioma. Craniotomy scar (white arrow). 
basilar invagination due to softening of bone affected by CFFD in the occiput (Figure 5) may give rise to symptoms of cranial neuropathy or brain stem compression and will also require monitoring in the future.

Our third case is an example of an iatrogenic complication of CFFD due to difficulty with interpretation of plain skull and facial radiographs in the years prior to the availability of CT scanning. The sclerotic lesion seen in the left sphenoid ridge (Figure 6) was considered suspicious of a hyperostotic meningioma but biopsy done at left temporal craniotomy revealed only abnormal fibrous tissue. CT done 20 years later (Figure 7) showed sclerotic bone thickening extending from the sphenoid into the adjacent frontal, zygomatic and pterygoid bones with no evidence of a meningeal mass. These appearances are typical of CFFD though differentiation from hyperostotic meningioma ('meningioma en plaque') or osteoma may be difficult, even with CT, especially in a monostotic lesion. ${ }^{13} \mathrm{Kim}$ et al..$^{13}$ recommended high resolution $\mathrm{CT}$ as the imaging technique of choice for the

\section{References}

1. McCune, D.J. Osteitis fibrosa cystica: the case of a nine year old girl who also exhibits precocious puberty, multiple pigmentation of the skin and hyperthyroidism. Am J Dis Child 1936, 52: 743-744.

2. Albright, F., Butler, A.M., Hampton, A.O. \& Smith, P. Syndrome characterised by osteitis fibrosa disseminata, areas of pigmentation and endocrine dysfunction, with precocious puberty in females: report of five cases. $N$ Engl J Med 1937, 216: 727-746.

3. Benedict, P.H. Endocrine features in Albright's syndrome (fibrous dysplasia of bone). Metabolism 1962, 11: 30-45.

4. Mauras, N. \& Blizzard, R.M. The McCune-Albright syndrome. Acta Endocrinol Suppl (Copenh) 1986, 279, 207-217.

5. Demner, L.P. Fibro-osseous lesions of bone. In: Ackerman, L.U., Spjut, H.J. \& Abell, M.R. (eds) Bone and Joints, International Academy of Pathology, Monograph No. 17. Baltimore, The Williams \& Wilkins Co., 1976, pp. 209-235.

6. Som, P. Fibrous dysplasia. In: Som, P.M. \& Bergeron, R.T. (eds.) Head and Neck Imaging, 2nd ed. St Louis, Mosby, 1991, pp. 208-210.

7. Valvassoni, G.E. \& Buckingham, R.A. Otosclerosis and bone dystrophies. In: Valvassoni, G.E., Buckingham, R.A., Carter, B.L., Hanafee, W.N. \& Mafee, M.F. (eds) Head and Neck Imaging. New York, Thieme, 1988, pp. 170-171. evaluation of such lesions while recognizing prob- 3 lems in making a firm diagnosis in some cases. $\stackrel{\mathbb{\Omega}}{\circ}$ However, in our case, CT would have allowed a $C$. definite diagnosis of CFFD if it had been available $\vec{F}$ at the time of initial presentation.

We conclude that CFFD has a wide spectrum of appearance and is often difficult to diagnose especially where it masquerades as or is complicated by other pathologies. The three cases presented in this paper illustrate rate manifesta- क tions or complications of CFFD, each of which $\vec{\circ}$ gave rise to diagnostic difficulty. CT was very useful for assessment of the pathological changes in all three cases. Its role in the management of such cases is unlikely to be reduced even with the increasing availability of MRI.

\section{Acknowledgements}

We thank Mr Thomas Fung and Ms Alice Orh for the illustrations.
8. Weber, A.L. Fibro-osseous lesions. In: Som, P.M. \& Bergeron, R.T. (eds) Head and Neck Imaging, 2nd edn. St Louis, Mosby, 1991, pp. 397-398.

9. Okudera, H., Toba, Y., Kyoshima, K. \& Kobayashi, S. Monostotic fibrous dysplasia of the sphenoid bone presenting with visual disturbance during pregnancy. No To Shinkei 1988, 40: 727-732 (English abstract).

10. Lipson, A. \& Hsu, T. McCune-Albright syndrome associated with acromegaly: report of a case and review of literature. Johns Hopkins Med J 1981, 149: 10-14.

11. Cuttler, L., Jackson, J.A., Uz-Zafar, M.S., Levitsky, L.L., Meltinger, R.C. \& Frohman, L.A. Hypersecretion of growth hormone and prolactin in McCune-Albright syndrome. $J$ Clin Endocrinol Metabol 1989, 68: 1148-1154.

12. Ameli, N. Fibrous dysplasia of the skull. Lancet 1955, 2: 480-482.

13. Kim, K.S., Rogers, L.F. \& Goldblatt, D. CT features of hyperostosing meningioma en plaque. Am J Roentgenol 1987, 149: $1017-1023$. 